\title{
Modular prostheses in the treatment of proximal humerus metastases: review of 40 cases
}

\author{
C. Scotti - F. Camnasio - G.M. Peretti - F. Fontana - G. Fraschini
}

Received: 23 October 2007 / Accepted: 14 January 2008 / Published online: 17 March 2008

\begin{abstract}
Background The humerus is the second most common site of metastatic bone disease involving long bones. Tumors which have a predilection for dissemination to bone are those of breast, prostate, thyroid, lung and kidney. The rationale for surgical treatment of these lesions is to prevent or treat pathological fractures in order to relieve pain and improve function.

Materials and methods Forty patients who had resection of the proximal humerus for metastatic bone disease and reconstruction with a modular prosthesis were retrospectively reviewed.

Results Mean functional outcome was $73.1 \%$ (Enneking score) and better results were achieved when a reverse prosthesis was implanted. Overall survival was $70 \%$ at 1 year, $42.5 \%$ at 2 years and $20 \%$ at 5 years. Local recurrence occurred in 4 patients, each of whom had initially been treated for a pathological fracture.

Conclusions It is important to follow rational guidelines, like those of Capanna and Mirels, in order to prevent
\end{abstract}

C. Scotti · F. Camnasio (西) · G.M. Peretti · F. Fontana - G. Fraschini Department of Orthopaedics and Traumatology

San Raffaele Scientific Institute

Via Olgettina 60, I-20132 Milan, Italy

e-mail: camnasio.francesco@hsr.it

\section{Scotti}

Residency Program in Orthopaedics and Traumatology I

Gaetano Pini Orthopaedic Institute

University of Milan, Milan, Italy

G.M. Peretti

Faculty of Exercise Sciences

University of Milan, Milan, Italy pathological fractures and to give the patient a definitive treatment, as the advances in the management of cancer prolong the survival of these patients. In this series, satisfactory results were obtained, giving the patients an acceptable quality of life.

Keywords Bone metastases · Proximal humerus · Modular prostheses $\cdot$ Pathological fracture

\section{Introduction}

Bone metastases are the most common example of bone malignancies and they largely exceed in number primary tumors of the skeleton. Bone metastatic disease strongly affects the survival of patients with cancer: in fact, the prognosis for patients with metastases ranges from fair to poor. Patients with lung carcinoma or melanoma that has metastasized to the skeleton generally have less than a 6month median survival; patients with bone metastases from thyroid and prostate carcinoma present a 45-month median survival [1].

The humerus is the second most common site of metastatic disease involving long bones. Humeral lesions may cause pain, disability and pathological fractures [2]. Untreated pathological fractures involving the extremities are generally responsible for serious disability. Additionally, the risk of fracture is different in lower and upper extremities. In fact, the bones of the lower limbs are subject to weight bearing, which makes $50 \%$ destruction a critical limit for impending fractures [3]. The risk of fractures is certainly lower in the upper limbs. Nevertheless, important intrinsic and extrinsic characteristics involving certain districts, such as the proximal humerus, should be considered as risk factors for spontaneous fractures. 
These are the largely cancellous structure of the metaphysis with low cortical rigidity and the significant rotational and bending forces to which this structure is subject [4]. In this regard, the definition of impending fracture is controversial: when destruction is greater than $75 \%$ of the bone's cross section, the risk of spontaneous fracture is great [5]. On the other hand, it should also be considered that, when only one cortex remains, the humerus often fractures with activities of daily living [5].

There are different treatment options for bone metastases. Chemotherapy and radiation therapy are appropriate treatments for sensitive tumors [6]. Bisphosphonates are useful in decreasing skeletal complications from breast and prostate carcinoma and multiple myeloma [7]. Surgery is indicated for intractable pain and for impending or established pathological fractures [8]. Conventional surgical strategies for humeral metastases are intramedullary nails, plate fixation and cement augmentation for reconstruction of large bone defects. Methylmethacrylate is also an effective adjuvant, both for improving the fixation with intramedullary nails or plates and for sterilizing the diaphysis from metastatic microfoci. On the other hand, when the lesion involves the proximal humerus, the choice for the optimal surgery is often difficult, especially when the destruction area involves the epiphysis.

However, advances in chemotherapy and local radiation techniques have led to an improvement in survival of these patients, and prolonged life expectancy can result in local progression of disease and failure of conventional fixation devices [9]. Therefore, a more radical and definitive treatment with prostheses seems to be indicated for these patients [10]. In the literature, indications for prosthetic replacement in patients with bone metastases are isolated lesions, extensive bone loss, and failed conventional reconstruction with plates or rods [4, 10-12]. Modular prostheses permit an adequate resections and restoration of acceptable functionality. Moreover, in case of isolated metastatic lesions, a wide resection and the implantation of a modular prosthesis may also be indicated $[4,10,12-16]$.

This paper reports our experience with prosthetic replacement in the treatment of proximal humeral metastases between 1992 and 2006.

\section{Materials and methods}

We retrospectively analyzed patients treated in our department with prostheses between 1992 and 2006 for proximal humeral metastases. Patients were considered eligible to receive a prosthesis if they had single metastatic lesions, impending fractures, pathological fractures or intractable pain not responsive to medical therapy and to radiation. Exclusion criteria were multiple osteoblastic lesions, osteolytic lesions with no risk of fracture and expected survival less than 2 months.

Indications for surgery in this retrospective series were compared with the protocol proposed by Capanna and Campanacci (Table 1) [4]. This protocol provides rational guidelines for indications for surgery, the type of operation to be undertaken and the methods of reconstruction available. Based on these criteria, patients in classes 1, 2 and 3 undergo surgical treatment when possible and have adjuvant treatment, if indicated. Patients in class 4 are initially referred for conservative treatment.

To evaluate the risk of pathological fractures, we used the scoring system proposed by Mirels [17], which is based on site, pain level, nature of the lesion, and size of the lesion relative to the diameter of the bone (Table 2). We considered mandatory the surgical treatment of pathological fractures with a score of 9 or higher, while a score of 8 required further clinical judgment, as described by others [9].

Functional outcome was assessed using the Musculoskeletal Tumor Society (MSTS) scoring system (Enneking score) for the evaluation of major skeletal resections and reconstructions; this score assesses pain, function, emotional acceptance, hand positioning, manual dexterity and lifting ability [18]. Oncologic outcome was evaluated by considering survival, local recurrence and need for revision surgery or adjuvant therapies.

In all patients, resection was done using a deltopectoral approach, which permits a wide view of the anterosuperior area of the proximal humerus. It allows for a wide resection of the proximal humerus, including the

Table 1 Summary of the management protocol proposed by Capanna and Campanacci [4]

\begin{tabular}{|c|c|}
\hline Class & Description \\
\hline 1 & $\begin{array}{l}\text { Solitary metastatic lesion } \\
\text { Primary tumor with good prognosis } \\
\text { Interval over } 3 \text { years since detection of the primary tumor }\end{array}$ \\
\hline 2 & Pathological fracture at any site \\
\hline 3 & Impending fracture in a major long bone \\
\hline 4 & $\begin{array}{l}\text { Osteoblastic lesions at all sites } \\
\text { Osteolytic or mixed lesions in non-structural bones (fibula, } \\
\text { rib, sternum, clavicle) } \\
\text { Osteololytic lesion with no impending fracture }\end{array}$ \\
\hline
\end{tabular}

Table 2 Assessment of the risk of pathological fracture according to Mirels [17]

\begin{tabular}{llll}
\hline Variable & 1 point & 2 points & 3 points \\
\hline Site & Upper limb & Lower limb & Peritrochanter \\
Pain & Mild & Moderate & Weight bearing \\
Lesion & Blastic & Mixed & Lytic \\
Size related to & $<1 / 3$ & $1 / 3-2 / 3$ & $>2 / 3$ \\
$\quad$ bone diameter & & & \\
\hline
\end{tabular}


entire subacromial bursa and the rotator cuff [19]. Metastatic lesions were usually resected by subperiosteal dissection, and the rotator cuff tendons were saved, if not involved by the metastatic disease during surgical exploration or at pre-operative magnetic resonance imaging (MRI). Modular prostheses were implanted by the same surgical equipe in our department. The prostheses were usually cemented in order to sterilize any metastatic microfoci located in the humeral shaft and to minimize the risk of pathological periprosthetic fractures.

\section{Results}

Between 1992 and 2006, we treated 40 patients (19 women, 21 men) for proximal humerus metastases. At the time of surgery, the patients had a mean age of 67 years (range, 52-75). The predominant primary tumor was breast carcinoma (13 patients), followed by renal cell carcinoma (12 patients), lung carcinoma (6 patients), prostatic carcinoma (5 patients), 2 lesions of unknown primary tumor, and plasmocytoma and melanoma (1 case each). Plasmocytoma was included since its biological behavior and the mechanical implications are similar to those of metastatic disease [4]. In 39 patients, the humerus alone was affected and in 1 case both the humerus and the scapula had metastases. Metastases were detected 6 months to 13 years after the diagnosis of the primary tumor in 32 patients $(80 \%)$, while in 8 patients $(20 \%)$ the metastasis was the first sign of the malignancy.

In 12 patients $(30 \%)$ surgery was performed to treat a pathological fracture (Capanna class 2). In the remaining 28 patients $(70 \%)$, prosthetic replacement was the primary treatment of the lesion, and was indicated for an impending fracture in 18 patients (class 3), for intractable pain in 5 patients (class 4 ), and for solitary lesions in 5 patients (class 1$)$. Overall, 10 patients $(25 \%)$ were admitted to surgery after local radiation therapy had been performed: 5 experienced a pathological fracture. Following surgery, 10 patients $(25 \%)$ were treated with local radiation therapy, 15 patients $(37.5 \%)$ were treated with chemotherapy and 8 patients $(20 \%)$ were treated with immunotherapy.

The patient with both humeral and scapular involvement underwent a modified Tikhoff-Linberg procedure: en bloc resection of the glenohumeral joint after an extracapsular osteotomy of the neck of the scapula, followed by reconstruction with a modular prosthesis [20] for aesthetic purposes only. In this case we used an I.O.R. modular prosthesis (Officine Ortopediche Rizzoli, Bologna, Italy) (Fig. 1) [21]. In 35 patients, we implanted an RPS endoprosthesis [22] (Lima-Lto, San Daniele del Friuli, Udine, Italy) (Fig. 2) and, in the last 4 patients treated, we used an SMR

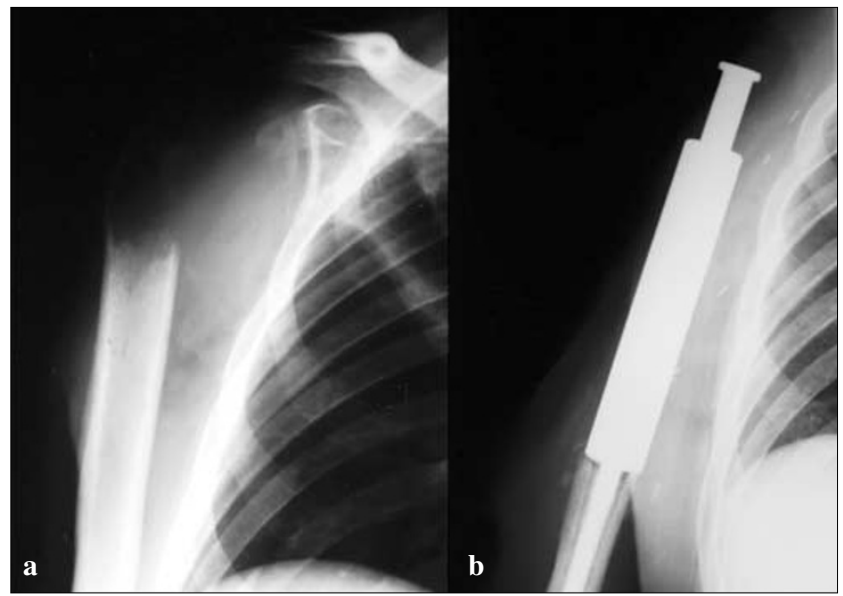

Fig. 1a,b A 65-year-old man with lung carcinoma. a Metastasis involving both the proximal humerus and the scapula. b He underwent modified Tikhoff-Linberg procedure and an I.O.R. prosthesis was implanted. No local recurrence occurred. Survival was 1 year; death occurred for visceral dissemination

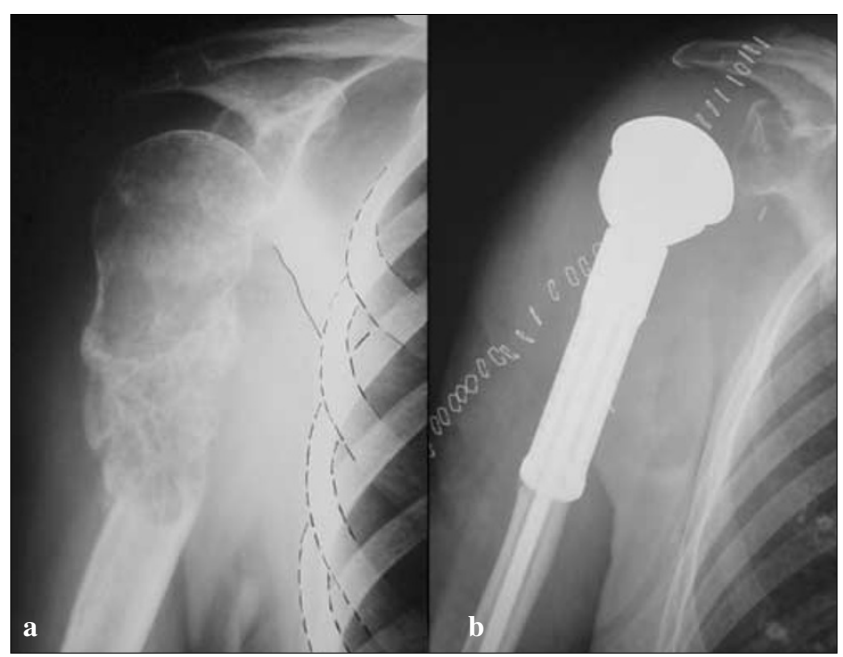

Fig. 2a,b A 72-year-old man with renal cell carcinoma. a Metastasis in the proximal humerus, initially treated with radiation therapy, caused a pathological fracture. b Treatment with a modular RPS prosthesis. Survival was 1 year; death occurred for visceral dissemination

reverse modular prosthesis (Fig. 3) (Lima-Lto). Three patients were lost to follow-up and the remaining 37 were followed for a mean of 2.5 years (range, 0.5-12 years).

Functional outcome was evaluated in 36 patients (90\%); it was not evaluated in the patient who received an I.O.R. modular prosthesis (SMR) for aesthetic reconstructive purposes only. The overall mean value of the Enneking score was $73.1 \%$ (95\% CI, $69.9 \%$ to $76.3 \%$ ). For patients with a reverse prosthesis (SMR) and for those with an anatomical prosthesis (RPS) mean scores were $87.5 \%$ (95\% CI, $82.7 \%$ to $92.3 \%$ ) and $71.3 \%$ (95\% CI, $68.2 \%$ to $74.4 \%$ ), respectively.

Oncologic outcome was evaluated in 37 patients and mean survival was 24.4 months (95\% CI, 19 to 29.8 months). Local recurrence-free survival was 23.1 months 


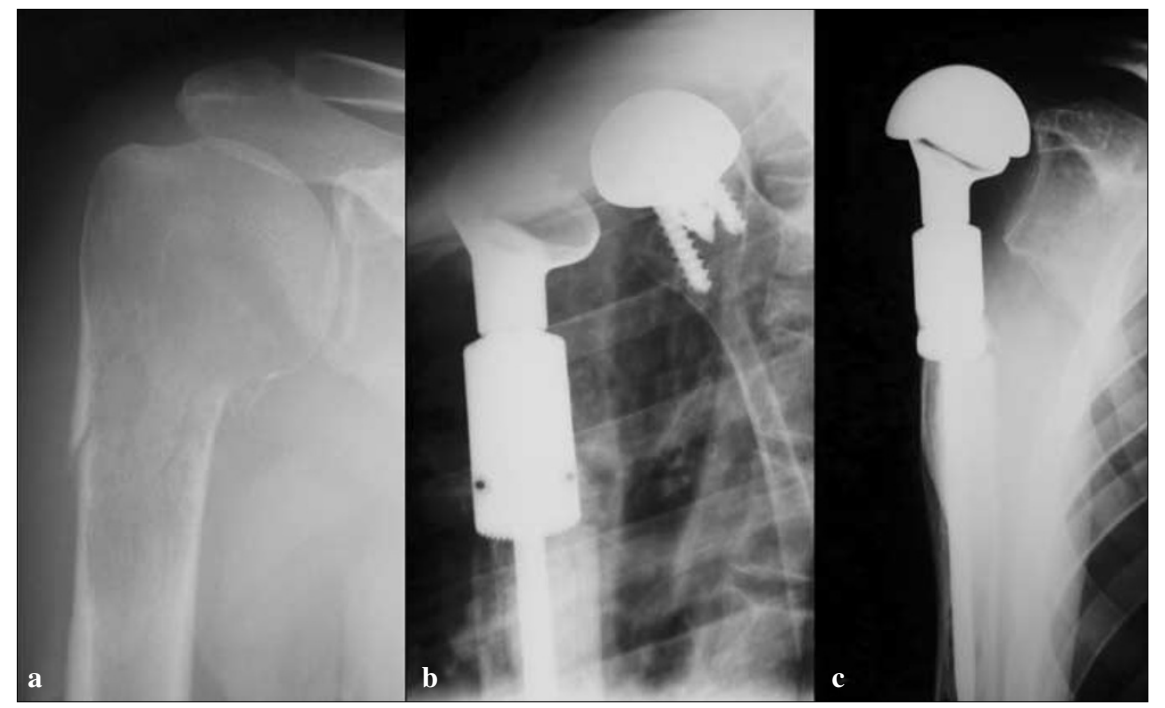

Fig. 3a-c A 52-year-old man with prostatic carcinoma. a Pathological fracture of the humerus. b After implantation of an SMR reverse prosthesis, local radiation therapy caused glenosphere loosening and dislocation of the prosthesis. c Revision surgery involved changing the humeral head with a CTA head. The patient is still alive with a follow-up of 4 years
Fig. 4 Kaplan-Meier plots of overall survival and local recurrence-free survival. Local recurrences presented between 6 and 12 months after implantation

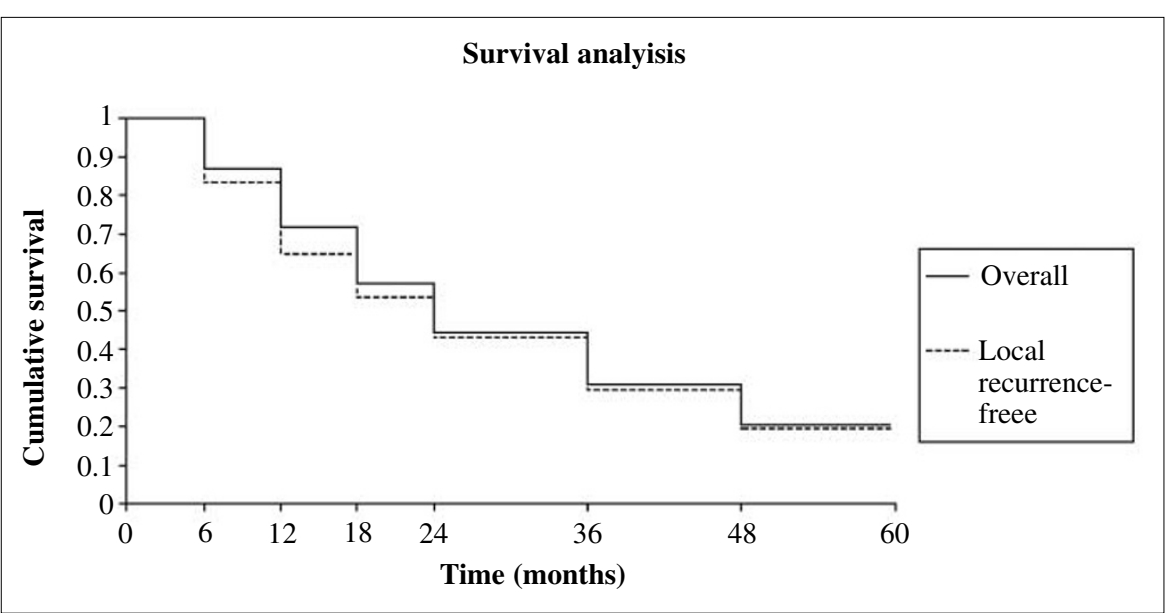

(95\% CI, 17.5 to 28.7 months). Overall, $70 \%$ of patients survived for 1 year after surgery, $42.5 \%$ survived for 2 years and $20 \%$ survived for 5 years or more (Fig. 4). Four patients presented a local recurrence between 6 and 12 months after surgery: the primary tumor was renal cell carcinoma in 2 cases, breast carcinoma in 1 case, and lung carcinoma in 1 case. Interestingly, all of them experienced previously a pathological fracture. They were then treated with local radiation therapy.

We noticed a few complications. Two patients experienced an early superficial wound infection, treated successfully with antibiotic therapy. No deep infections were reported. Superior dislocation of the humeral head occurred in 3 cases, when sparing the rotator cuff was not possible, and an RPS prosthesis was implanted: revision surgery was performed by changing the humeral head with a CTA (Cuff Tear Arthropathy) head (Lima-Lto). Glenosphere loosening and dislocation of the prosthesis occurred in one patient who underwent adjuvant local radiation therapy: revision surgery was performed by changing the humeral head with a CTA head (Fig. 3) (Lima-Lto).

\section{Discussion}

The aim of surgical treatment of bone metastases is to prevent or stabilize pathological fractures, relieve pain, avoid local recurrence and permit a satisfactory functionality of the operated limb in order to achieve an acceptable quality of life. Short-term improvement in pain and functional status seems particularly desirable for patients with limited life expectancy [6]. Moreover, operative treatment for pathological fractures of the humerus is generally superior to nonoperative methods of fracture management such as splints or casts [23]. As a matter of fact, Flemming and Beals demonstrated non-union in 50\% of cases and inadequate pain control in $88 \%$ of patients treated nonoperatively [23].

Pathological fracture always requires an extended time to heal, and as many as $50 \%$ will never heal at all [24]. Moreover, radiation therapy used to treat tumor pain can delay fracture healing. Biomechanical features of the proximal humerus are slightly different from those of the lower limb, because of the absence of weight bear- 
ing. Nevertheless, there are important rotational and bending forces due to the action of the rotator cuff, deltoid, pectoralis maximum and latissimus dorsi. Additionally, the metaphysis is largely cancellous with low cortical rigidity; the absence of strong cortical structures frequently leads to extracompartmental spreading of the metastatic lesions. For these reasons, osteosynthesis is no longer recommended for metastases in this site, while the use of modular endoprostheses permits adequate resection with acceptable oncologic and functional results [4]. Moreover, especially in tumors with a poor response to radiotherapy, the incidence of complications is lower after implantation of an endoprosthesis than after osteosynthesis [25].

In this series, the I.O.R. modular prosthesis [21] allowed for radical resection, such as the modified TikhoffLinberg procedure [20], but it did not permit articular reconstruction. The RPS prosthesis permitted to achieve acceptable functional results while performing radical resections, when it was possible to spare the rotator cuff and deltoid. On the other hand, when it was mandatory to resect the rotator cuff, the functional result was usually unsatisfactory. Moreover, the aim of sparing soft tissues could result in an inadequate non-radical treatment.

The SMR modular system, characterized by high modularity features, permits personalization of the treatment. When it is possible to spare the rotator cuff, it allows for the implantation of conventional endoprostheses; when excision of the rotator cuff, capsule and ligaments is mandatory, a reverse prosthesis can be implanted. With a reverse prosthesis, it is possible to achieve good functional results, even in case of wide resections, if the deltoid insertion is preserved. In reverse prostheses, the humerus is converted into a socket and the glenoid to a ball, providing a stable fulcrum for glenohumeral articulation [26]. This is achieved through maximization of the length-tension relationship of the deltoid and remaining cuff musculature [27]. In case of detachment of the glenosphere due to bone loosening, SMR system permits substitution of the head with a CTA head, which has a larger articular area that allows the head to fit into a socket formed by the glenoid and the acromion. When deltoid activation raises the arm, the head surface remains in contact with the acromion for a longer time, which increases the amount that the arm can be raised by this muscle. Also in this case, preservation of the deltoid insertion is mandatory for obtaining satisfactory results. Results are also dependent on the patient's endurance, strength and participation in rehabilitation [5]. When adjuvant local radiation therapy is performed, there is a high risk of glenosphere loosening as highlighted by one of our cases. Consequently, we no longer implant reverse prostheses when radiation therapy is planned.
We believe that the risk of pathological fracture is a more important requirement for surgery than supposed life expectancy. In fact, all patients who had a local recurrence had previously had a pathological fracture, which may have favored spreading of the tumor in the soft tissues. Obviously, life expectancy should be longer than recovery from surgery $[6,28,29]$. In order to preserve the quality of life of these patients, surgery should be definitive [30] and the use of prostheses allows wide resections of the lesions. This is also a useful technique when radiochemotherapy cannot be performed.

This study is limited by the fact that it is a retrospective analysis of heterogeneous group of patients, without a control group, a preoperative assessment or a qualityof-life evaluation. Nevertheless, it offers some interesting information. Our current approach to bone metastases, in view of the results of this study and the literature, is based on Capanna's criteria [4]. In this series, satisfactory functional results were achieved with few complications. The aim of this treatment is to give these patients an acceptable quality of life, independently from life expectancy.

Conflict of interest statement The authors declare that they have no conflict of interest related to the publication of this manuscript.

\section{References}

1. Coleman RE (1997) Skeletal complications of malignancy. Cancer 80[Suppl 8]:1588-1594

2. Bashore CJ, Temple HT (2000) Management of metastatic lesions of the humerus. Orthop Clin North Am 31(4):597-609

3. Hipp JA, Springfield DS, Hayes WC (1995) Predicting pathologic fracture risk in the management of metastatic bone defects. Clin Orthop Relat Res 312:120-135

4. Capanna R, Campanacci DA (2001) The treatment of metastases in the appendicular skeleton. J Bone Joint Surg Br 83(4):471-481

5. Frassica FJ, Frassica DA (2003) Evaluation and treatment of metastases to the humerus. Clin Orthop Relat Res 415[Suppl]:S212-S218

6. Talbot M, Turcotte RE, Isler M et al (2005) Function and health status in surgically treated bone metastases. Clin Orthop Relat Res 438:215-220

7. Mundy GR, Yoneda T (1998) Bisphosphonates as anticancer drugs. N Engl J Med 339(6):398-400

8. Rougraff B (2000) Indications for operative treatment. Orthop Clin North Am 31(4):567-575

9. Jacofsky DJ, Haidukewych GJ (2004) Management of pathologic fractures of the proximal femur: state of the art. J Orthop Trauma 18(7):459-469

10. Eckardt JJ, Kabo JM, Kelly CM et al (2003) Endoprosthetic reconstructions for bone metastases. Clin Orthop Relat Res 415[Supp1]:S254-S262

11. Frassica FJ, Frassica DA (2003) Metastatic bone disease of the humerus. J Am Acad Orthop Surg 11(4):282-288

12. Fuchs B, Trousdale RT, Rock MG (2005) Solitary bony metastasis from renal cell carcinoma: significance of surgical treatment. Clin Orthop Relat Res 431:187-192 
13. Bickels J, Kollender Y, Wittig JC et al (2005) Function after resection of humeral metastases: analysis of 59 consecutive patients. Clin Orthop Relat Res 437:201-208

14. Schulte M, Hartwig E, Sarkar M, Arand M (1998) Endoprosthetic treatment of metastatic pathological fractures. Anticancer Res 18(3C):2251-2252

15. Wedin R (2001) Surgical treatment for pathologic fracture. Acta Orthop Scand Suppl 72(302):1-29

16. Camnasio F, Ravasi F (1996) Modular prostheses in metastatic bone disease of the proximal femur. Bull Hosp Jt Dis 54(4):211-214

17. Mirels H (1989) Metastatic disease in long bones. A proposed scoring system for diagnosing impending pathologic fractures. Clin Orthop Relat Res 249:256-264

18. Enneking WF, Dunham W, Gebhardt MC et al (1993) A system for the functional evaluation of reconstructive procedures after surgical treatment of tumors of the musculoskeletal system. Clin Orthop Relat Res 286:2416

19. De Wilde LF, Plasschaert FS, Audenaert EA, Verdonk RC (2005) Functional recovery after a reverse prosthesis for reconstruction of the proximal humerus in tumor surgery. Clin Orthop Relat Res 430:156-162

20. Capanna R, van Horn JR, Biagini R et al (1990) The TikhoffLinberg procedure for bone tumors of the proximal humerus: the classical "extensive" technique versus a modified "transglenoid" resection. Arch Orthop Trauma Surg 109(2):63-67

21. Capanna R, Mapelli S, Ruggieri P et al (1988) Resection of the proximal humerus and I.O.R. modular prosthesis in the treatment of metastatic lesions. Ital J Orthop Traumatol 14(2):143-148

22. Ippolito V, Saccalani M, Ianni L et al (2003) Resection of the proximal humerus for metastases and replacement with RPS prosthesis. Chir Organi Mov 88(2):165-69

23. Flemming JE, Beals RK (1986) Pathologic fracture of the humerus. Clin Orthop Relat Res 203:258-60

24. Gainor BJ, Buchert P (1983) Fracture healing in metastatic bone disease. Clin Orthop Relat Res 178:297-302

25. Wedin R, Bauer HC, Wersäll P (1999) Failures after operation for skeletal metastatic lesions of long bones. Clin Orthop Relat Res 358:128-39

26. Grammont PM, Baulot E (1993) Delta shoulder prosthesis for rotator cuff rupture. Orthopedics 16(1):65-68

27. Frankle M, Siegal S, Pupello D et al (2005) The Reverse Shoulder Prosthesis for glenohumeral arthritis associated with severe rotator cuff deficiency. A minimum two-year follow-up study of sixty patients. J Bone Joint Surg Am 87(8):1697-1705

28. Damron TA (2002) Management of metastatic carcinoma to the hip and proximal femur. In: Mendez LR (ed) Orthopaedic knowledge update: musculoskeletal tumors. American Academy of Orthopaedic Surgeons, Rosemont, pp 363-371

29. Parrish FF, Murray JA (1970) Surgical treatment for secondary neoplastic fractures. A retrospective study of ninety-six patients. J Bone Joint Surg Am 52(4):665-686

30. Bohm P, Huber J (2002) The surgical treatment of bony metastases of the spine and limbs. J Bone Joint Surg Br 84(4):521-529 